\title{
Microcotyle donavini Van Beneden et Hesse 1863 espèce type du genre Microcotyle Van Beneden et Hesse 1863
}

\author{
Par Louis EUZET et Annie MARC
}

Microcotyle donavini Van Beneden et Hesse 1863 est considéré par N. G. Sproston comme l'espèce-type du genre Microcotyle Van Beneden et Hesse 1863. N. G. Sproston discute d'ailleurs dans sa monographie de 1946 les raisons pour lesquelles elle emploie l'orthographe « donavini » au lieu de * donavani » de la publication originale de Van Beneden et Hesse. Elle indique aussi pourquoi elle suit Stiles et Hassal et choisit Microcotyle donavini comme espèce-type du genre. Nous nous rallions à ces conclusions.

Microcotyle donavini est un Monogenea Polyopisthocotylea commun sur les branchies de Labrus bergylta Ascan. Ce Labre vit dans la Manche et la Mer du Nord et sur les côtes britanniques de l'Atlantique.

Microcotyle donavini n'est actuellement connu que par:

1) La description originale succincte de Van Beneden et Hesse. Cette description qui porte uniquement sur la morphologie externe est accompagnée de 11 figures (PI. XII, fig. 1 à 11);

2) Le travail de Scott (1905) avec 1 figure ;

3) La révision de Sproston (1946) qui donne avec une courte description de la morphologie externe des détails sur l'anatomie. Cinq dessins (fig. 101, $a$ à $e$ ) illustrent ce texte.

Cela nous a paru insuffisant pour le type d'un genre qui compte actuellement près de 100 espèces. Nous avons donc repris l'étude de Microcotyle donavini afin d'en donner une description morphologique et anatomique détaillée.

Le matériel qui a servi à cette révision a été recueilli à Roscoff (Finistère) durant le mois de juillet 1962 sur les branchies de Labrus bergylta Ascan. Nous étudierons plus loin la répartition et la localisation du parasite sur l'hôte. 

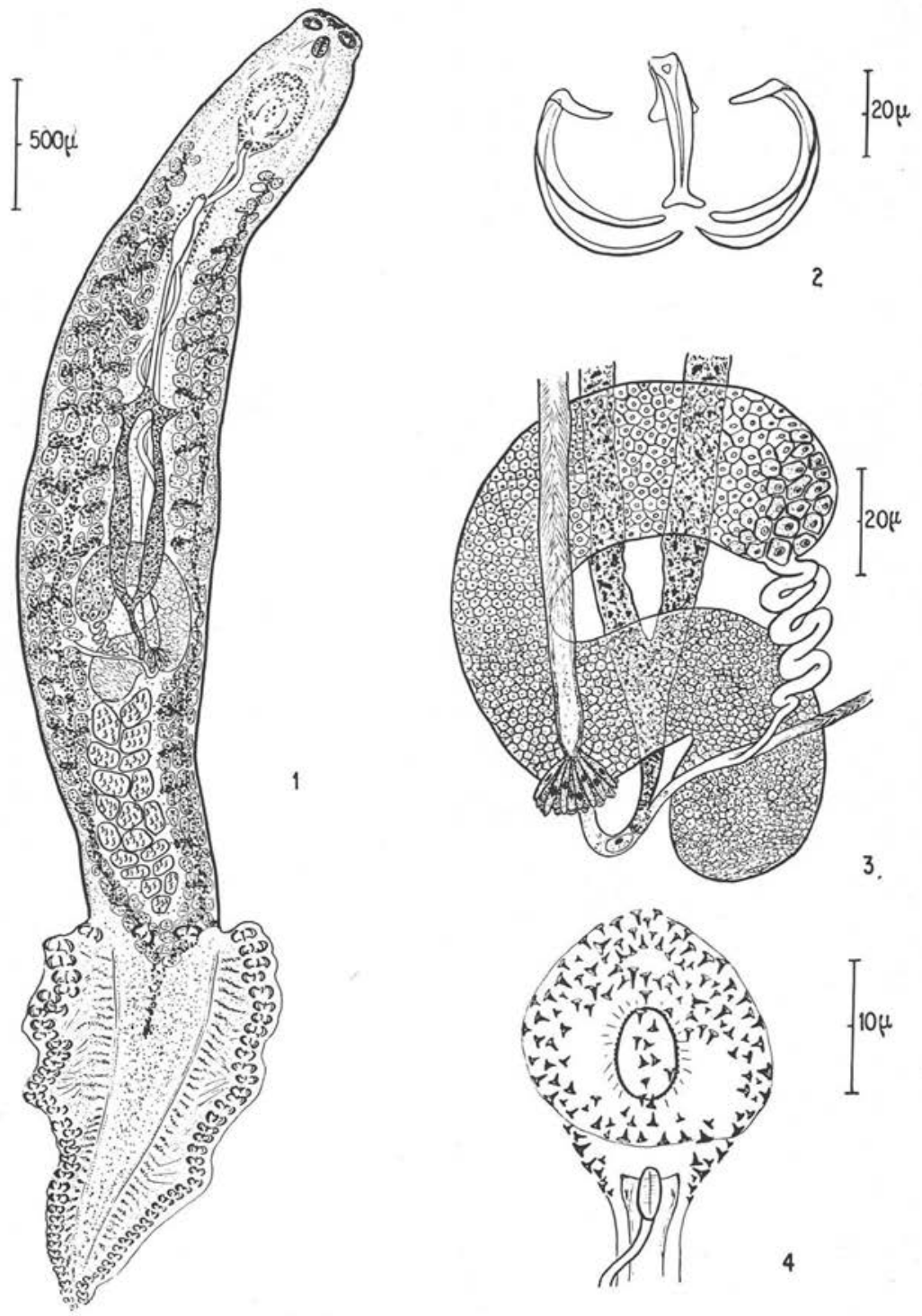

Microcotyle donavini Van Beneden et Hesse, 1863

FIG. 1. - Vue dorsale d'après une préparation in toto

FIG. 2. - Sclérites d'une pince, vue antérieure

FIG. 3. - Complexe génital, détail, vue ventrale

Fig. 4. - Atrium génital, répartition des épines, vue ventrale d'après une préparation aplatie 
Microcotyle donavini Van Beneden et Hesse 1863.

Hôte: Labrus bergylta Ascan.

Habitat : Branchies.

Localité : Roscoff (Finistère).

\section{Morphologie externe :}

Microcotyle donavini est un petit Monogène de 4 à $5 \mathrm{~mm}$ de long et de 0,4 à $0,5 \mathrm{~mm}$ de large. Le ver présente deux parties : le corps proprement dit $(3 \mathrm{~mm})$ légèrement rétréci au niveau du quart antérieur et le hapteur $(1 \mathrm{~mm})$ triangulaire à pointe postérieure (fig. 1).

A l'extrémité antérieure s'ouvre la bouche subterminale ventra!e. En arrière, entre la bouche et l'étranglement antérieur, on distingue sur la ligne médio-ventrale l'ouverture de l'atrium génital. Le vagin débouche dorsalement au milieu de l'étranglement.

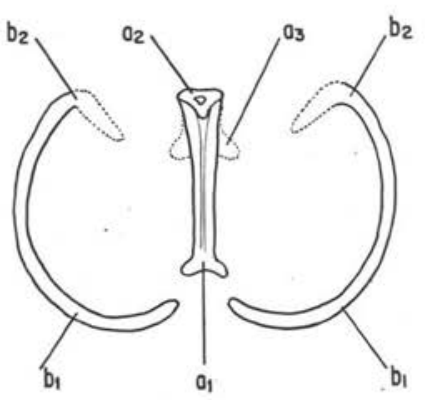

5

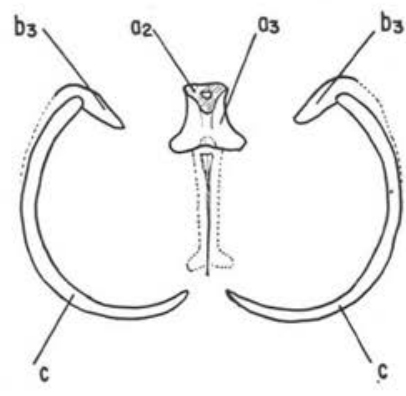

6

Microcotyle donavini Van Beneden et Hesse, 1863 - Pince

FIG. 5. - Disposition des sclérites dans la mâchoire antérieure

FIG. 6. - Disposition des sclérites dans la mâchoire postérieure

A l'arrière le hapteur porte de chaque côté une rangée de pinces comportant chacune deux mâchoires soutenues par des pièces sclérifiées ou sclérites (fig. 2). En moyenne nous en avons compté 43 paires disposées symétriquement.

La mâchoire antérieure (fig. 5) de chaque pince présente sur la ligne médiane une pièce sclérifiée $a_{1}$, terminée en $\mathrm{T}$ à branches courtes à son extrémité distale. Du côté proximal $a_{1}$ se replie en $a_{2}$ et passe dans la mâchoire postérieure. En coupe ce sclérite est grossièrement triangulaire à arête interne et à centre creux. Les bords de la mâchoire antérieure sont soutenues par deux pièces arquées $b_{1}$. Du côté distal ces pièces se terminent sur la ligne médiane au niveau de l'extrémité en $\mathrm{T}$ de $a$. Du côté proximal chacune de ces pièces $b_{1}$ se replie $b_{2}$ et donne dans la mâchoire postérieure une courte lame $b_{3}$.

La mâchoire postérieure (fig. 6) présente sur la ligne médiane la continuation $a_{3}$ de $a_{1} a_{2}$. Cette pièce élargie atteint le milieu de la mâchoire où elle se termine par deux expansions latérales arrondies. $a_{3}$ semble se prolonger sur la ligne médiane par une sclé- 
rification très mince. Les bords de la mâchoire postérieure sont soutenus par des sclérites arqués $c$ qui viennent s'articuler du côté proximal dans la courbe $b_{2}$. Du côté distal, les deux pièces $c$ se rapprochent sur la ligne médiane.

Du côté proximal un muscle puissant formant la paroi de la mâchoire relie de chaque côté $a_{2}$ à $b_{2}$. La disposition des sclérites telle que nous venons de la décrire doit être considérée comme la disposition caractéristique du genre Microcotyle. Cet arrangement ressemble beaucoup à celui des sclérites des pinces de Discocotyle sagittata (Leuckart 1842) décrit récemment par Llewellyn et Owen. Mais chez Discocotyle les pièces postérieures latérales $c$ sont subdivisées en deux pièces nommées par Llewellyn et Owen $c_{1}$ et $c_{2}$. Le mode de fonctionnement de ces deux types de pinces semble être identique.

Dans le hapteur toutes les pinces n'ont pas une taille unique, les postérieures sont en général plus petites que les antérieures.

Une moyenne calculée sur les pinces de 10 individus nous a donné :

- Pinces postérieures : $42 \mu$ de large et $33 \mu$ de long.

- Pinces antérieures : $87 \mu$ de large et $50 \mu$ de long.

\section{Anatomie interne :}

Antérieurement à la bouche, fait suite une cavité buccale où s'ouvre de chaque côté une ventouse musculaire ovoïde de $65 \mu$ de long et $40 \mu$ de large. Chaque ventouse est divisée en deux par un septum musculaire transverse. Le bord de ces ventouses buccales présente une rangée régulière de petites expansions coniques dont le nombre varie autour de 60. Extérieurement, entre l'extrémité antérieure du corps et la bouche se placent de chaque côté un groupe de glandes céphaliques.

Sur la ligne médiane, légèrement en arrière des ventouses, le pharynx se présente comme un barrilet de $50 \mu$ de long et $40 \mu$ de large qui débute dans la région dorsale de la cavité buccale. Sur coupes, on distingue deux parties, une supérieure musculaire à fibres rayonnantes et une inférieure glandulaire.

L'œsophage qui fait suite au pharynx prend naissance ventralement. D'abord simple il contourne dorsalement l'atrium génital, puis se bifurque et donne les deux longues branches latérales de l'intestin. Chaque branche présente sur son trajet de petits cœcums latéraux et axiaux. Les deux branches se rejoignent à la partie postérieure du corps pour former sur la ligne médiane, un diverticule unique qui pénètre dans la moitié antérieure du hapteur.

\section{SYSTÈME GÉNITAL :}

Appareil mâle: Les testicules dont le nombre varie de 18 à 22 sont des masses ovoïdes aplaties dorsoventralement et situées dans la moitié postérieure du corps. Leur diamètre apparent varie de 83 à $58 \mu$. Les testicules antérieurs sont les plus gros et la taille va en diminuant vers l'extrémité postérieure. Le canal déférent, légèrement sinueux remonte suivant la ligne médio-longitudinale du corps. Il aboutit à l'extrémité d'une papille située à la partie inférieure de l'atrium génital. 
Vu par la face ventrale cet atrium se présente comme une région ovalaire de $250 \mu$ de long et $175 \mu$ de large disposée longitudina'ement et entourée d'une zone de fibres musculaires radiaires. L'ouverture ventra'e est circulaire variable, mais relativement petite. Les parois de cet atrium sont irrégulièrement couvertes de petites épines qui ont, en vue ventrale, la disposition suivante (fig. 4) : à l'avant un amas en couronne entoure une zone musculaire presque dépourvue d'épines ; au-dessous, une région inter-

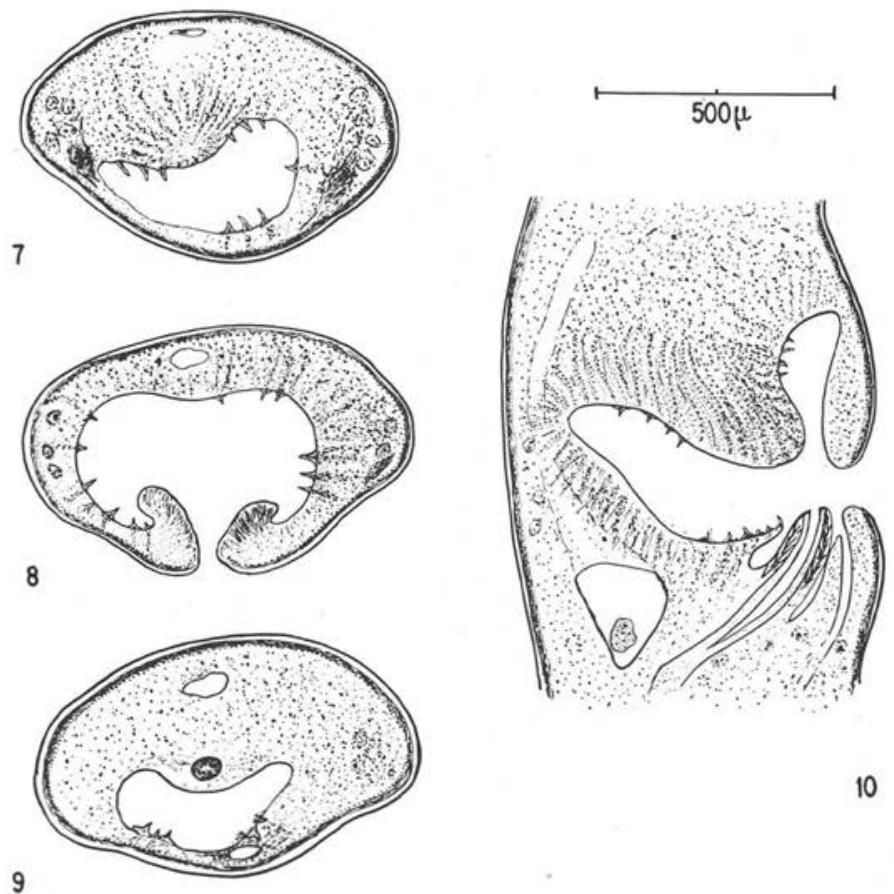

Microcotyle donavini Van Beneden et Hesse, 1863

FIG. 7, 8, 9. - Coupes transversales à différents niveaux de l'atrium génital

FIG. 10. - Coupe sagitale passant par le canal déférent et l'utérus

médiaire présente de chaque côté une bande latérale en arc de cercle, et au centre, une bande médiane ; à l'arrière de petits champs épineux dessinent un $\mathrm{V}$ qui flanque les ouvertures mâles et utérines.

L'atrium génital se présente en coupe comme une large cavité où vers l'avant un bulbe musculeux fait une saillie axiale (fig. 7-10). Ce bulbe détermine autour de lui un sillon circulaire. Les parois du bulbe et du sillon sont couvertes d'épines (fig. 7-10). Celles-ci forment en vue ventrale la couronne antérieure signalée plus haut. Les parois dorsales et latérales de la cavité sont couvertes d'épines. 
Sur la face ventrale l'ouverture de l'atrium est flanquée par deux champs musculaires (fig. 8).

Sur la face postérieure s'ouvrent le pore mâle à l'extrémité d'une papille et l'utérus ventral. Le canal déférent et l'utérus sont séparés par une invagination de l'atrium, invagination dont les parois latéra!es portent un petit groupe d'épines (fig. 9).

Examinées à un fort grossissement, les épines de l'atrium présentent une base circulaire élargie supportant une pointe creuse courte et trapue. Les fibres musculaires qui entourent l'atrium semblent se rattacher aux bases circulaires des épines.

Appareil femelle : L'ovaire est situé au milieu du corps. Il débute postérieurement au niveau des premiers testicu'es, monte légèrement pour former une petite anse antérieure, puis passe sur le côté droit, remonte et en arc de cercle repasse sur le côté gauche. Là, il débouche dans l'oviducte ; cet oviducte très contourné descend et au niveau du début de l'ovaire se détache le canal génito-intestinal oui va déboucher dans la branche gauche de l'intestin (fig. 3). L'oviducte continue alors vers la droite du corps et sur la ligne médiane reçoit le vitelloducte impair. Il forme a'ors une anse postérieure qui se termine par une partie rétrécie entourée par la rosette des glandes de Mehlis. Au-delà, l'ootype sans différenciation nette se pro'onge par un utérus ventral presque rectiligne. Cet utérus cilié se termine dans l'atrium génital au-dessous de l'ouverture mâle.

L'ouverture vaginale dorsale est située à $750 \mu$ environ de l'extrémité antérieure soit le quart de la longueur du corps. Le vagin débute par un élargissement, à parois minces plissées. Cette poche vagina'e allongée longitudina'ement, est bordée à droite et à gauche d'un champ important de fibres musculaires dorso-ventrales groupées en faisceaux. Un rétrécissement marque la fin de cette poche vaginale.

Au-delà, le vagin se continue par un petit canal qui vient déboucher au point de rencontre des vitelloductes transverses (fig. 1).

Les glandes vitellogènes forment de chaque côté une large bande. Les deux bandes se réunissent dans la partie postérieure du corps en arrière des testicules. Ils forment alors une bande unique qui pénètre légèrement dans la partie axiale du hapteur. Les vitelloductes transverses se détachent à la moitié du corps environ et se réunissent sur la ligne médiane. Antérieurement ils reçoivent le vagin. En arrière, ils se séparent en deux vitelloductes latéraux qui se rejoignent à nouveau entre les deux parties transversales de l'ovaire pour donner le vitelloducte impair médian. C'est ce vitelloducte impair qui débouche dans l'oviducte. En vue dorsale ces vitelloductes bourrées de cellules vitellines brun foncé dessinent un ovale très allongé longitudinalement.

Les œufs fusiformes mesurent de 200 à $225 \mu$ de long et de 75 à $80 \mu$ de large. Ils présentent un filament polaire à chaque extrémité. L'un est court $(100 \mu)$ assez épais et se termine en crosse simple. A l'autre pôle le filament est long, mince et en général pelotonné sur lui-même (fig. 12).

\section{Développement :}

Les œufs placés dans de petits cristallisoirs remplis d'eau de mer renouvelée quotidiennement ont éclos au bout de 11 jours. L'oncomiracidium cilié sort par une ouver- 
ture circulaire qui prend naissance par rupture de la coque au 1/5 environ de la longueur de l'œuf. Le clapet ainsi formé se détache au pôle portant le long filament (fig. 12).

L'oncomiracidium est aplati dorsoventralement. Très variable dans ses dimensions, il mesure en extension moyenne $200 \mu$ de long et $70 \mu$ de large (fig. 11).
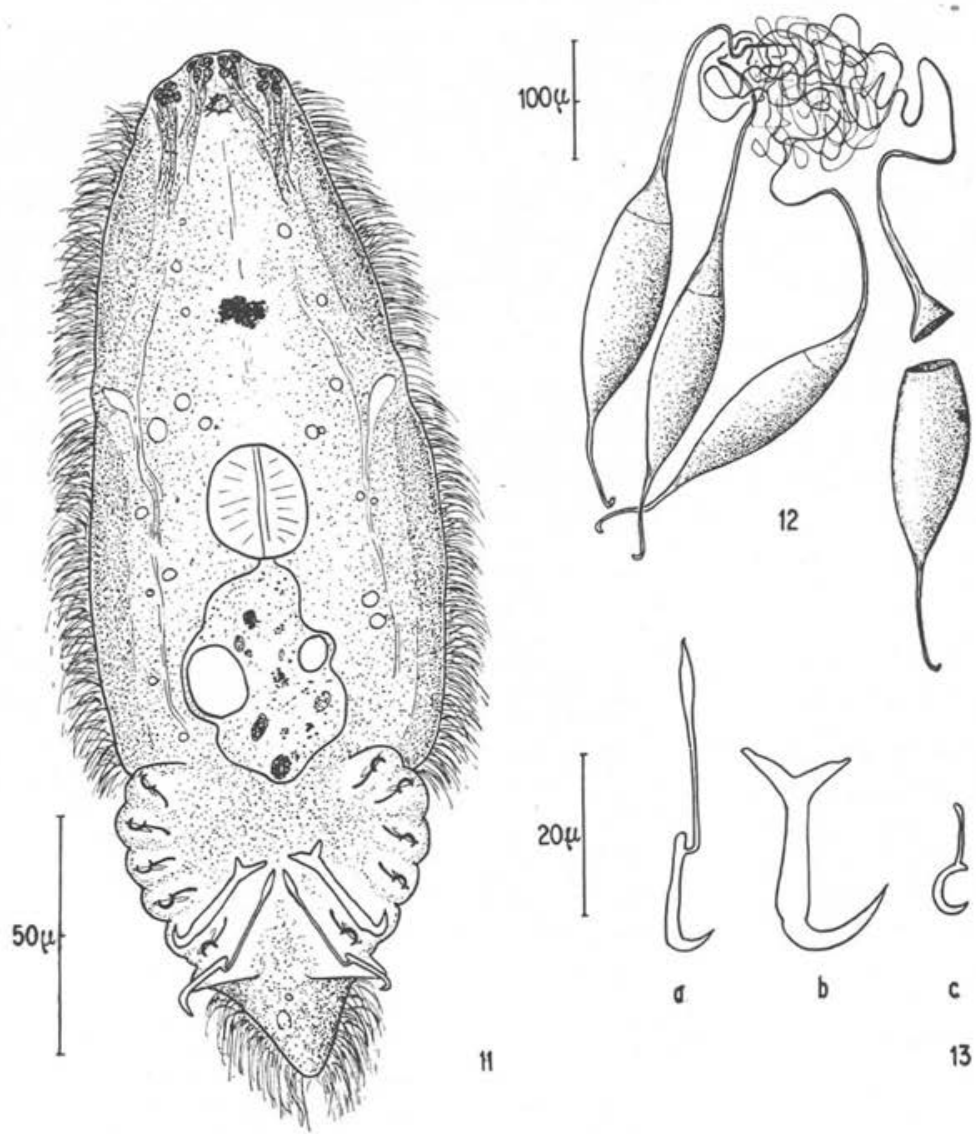

Microcotyle donavini Van Beneden et Hesse, 1863

FIG. 11. - Oncomiracidium, vue ventrale

Fig. 12. - Eufs et œuf éclos

FIG. 13. - Crochets du disque adhésif de l'oncomiracidium: a) crochet en fléau, postérieur, b) hamuli, c) crochet à conducteur

La ciliature s'étend comme une bande latérale de chaque côté, sauf à l'extrémité antérieure et sur une courte zone dorsale au niveau de la moitié du corps. Les bords du disque adhésif ne sont pas ciliés. A l'arrière le cône postérieur est complètement recouvert de cils. 
Les glandes céphaliques forment 3 paquets débouchant dans la région antérieure, 1 médian et 2 latéraux. Le paquet central peut être considéré comme la réunion sur la ligne médiane de deux groupes latéraux.

Sur la ligne médiane, à $50 \mu$ environ de l'extrémité antérieure on distingue la tache oculaire. C'est une petite zone irrégulière de pigment brun foncé.

Toujours sur la ligne médiane en arrière de la tache oculaire, on distingue le pharynx. Il se présente comme une masse musculaire sphérique de $20 \mu$ de diamètre. L'intestin sacciforme qui lui fait suite renferme de gros granules et un ou deux globules clairs plus importants.

Le système excréteur est formé de néphridies en nombre relativement réduit et de canalicules qui se rejoignent de chaque côté dans un canal latéral. Celui-ci débouche à l'extérieur au niveau des zones dorsales non ciliées situées à la moitié du corps.

L'armature du disque adhésif comprend:

-2 grands crochets postérieurs en fléau.

Le manche en baguette, légèrement élargi à l'extrémité proximale mesure $25 \mu$ de long. La lame en serpe s'articule d'une manière particulière à l'extrémité de la baguette (fig. 13 a). La lame mesure $15 \mu$ de long.

- 2 crochets postéro-latéraux à conducteur ; ils ont $12 \mu$ de long.

-2 hamulis de $30 \mu$ de long avec une lame de $18 \mu$, élargie légèrement avant de se recourber en une pointe de $12 \mu$. Le manche court $(8 \mu)$ a une longueur égale à celle de la garde, mais cette dernière est légèrement plus épaisse (fig. 13b).

-8 crochets latéraux à conducteur (4 de chaque côté). Ces crochets mesurent de 10 à $12 \mu$ (fig. 13c).

Sur un Labrus bergylta mort dans l'aquarium de la Station de biologie marine de Roscoff, nous avons recueilli toute une série de post-larves qui nous permettent d'affirmer que la suite du développement de Microcotyle donavini est semblable au développement des Microcotyle déjà étudiés, c'est-à-dire :

Microcotyle spinicirrus Mac Callum, 1918 par ReMLEY, 1942.

Microcotyle gotoï Yamaguti, 1934 par BYcHowsKY, 1957.

Microcotyle chrysophrii Van Beneden et Hesse, 1863 par Euzet, 1957.

Les pinces se forment autour des crochets latéraux dans le sens arrière-avant.

Nous devons cependant signaler une transformation de la morphologie des crochets en fléau et des hamulis. Chez une larve à 5 paires de pinces, les crochets larvaires postérieurs, postéro-latéraux et hamulis sont encore présents sur une languette postérieure médiane. Les crochets en fléau semblent légèrement plus longs, le manche s'est épaissi et paraît soudé à la lame en serpe. L'articulation caractéristique a disparu. Les hamulis sont un peu plus grands. La différence de taille provenant surtout de l'épaississement du crochet et de la morphologie légèrement différente du manche et de la garde (fig. 14). 
Comme chez Microcotyle chrysophrii la languette avec ses 6 crochets disparaît lorsque la larve possède 6 ou 7 paires de pinces.

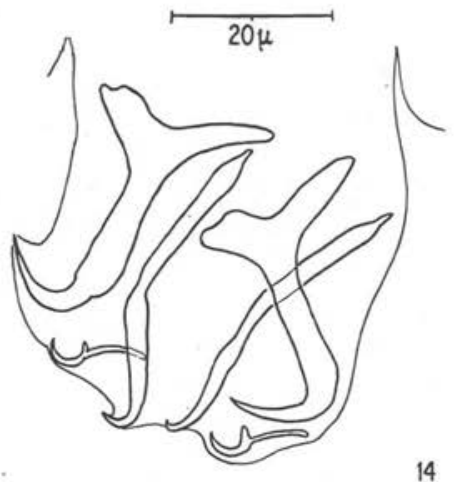

\author{
Microcotyle donavini Van Beneden et \\ Hesse, 1863
}

Fig. 14. - Languette postérieure avec crochets larvaires transformés chez une post-larve à 5 paires de pinces

14

Répartition et localisation de Microcotyle donavini sur Labrus bergylta.

Nous avons examiné 30 Labres dont la taille était comprise entre $7,5 \mathrm{~cm}$ et $60 \mathrm{~cm}$ de longueur totale. 21 Poissons étaient parasités ce qui donne un taux élevé de $70 \%$.

Nous indiquons dans le tableau ci-dessous le nombre de parasites selon la taille de l'hôte :

\begin{tabular}{|c|c|c|}
\hline TAILle Nombre & TAILle Nombre & TAille Nombre \\
\hline $7,5: 0$ & $16: 12-5$ & $22,5: 9$ \\
\hline $8: 0$ & $17: 5-4$ & $23,5: 0$ \\
\hline $8,5: 0$ & $18: 9-8-7$ & $24,5: 32-1$ \\
\hline $9: 0$ & $19,5: 5$ & $26: 4$ \\
\hline $10: 0$ & $20: 4$ & $27,5: 4$ \\
\hline $12: 0$ & $21 \div 8-0$ & $32: 14$ \\
\hline $14: 4$ & $21,5: 7$ & $38: 8$ \\
\hline $14,5: 15$ & $22: 0$ & $60: 22$ \\
\hline
\end{tabular}

Comme ce tableau le met en évidence nous n'avons jamais récolté de parasites sur des poissons de taille inférieure à $12 \mathrm{~cm}$. Au-dessus de cette taille le nombre de Microcotyle par hôte paraît très irrégulier. 
Sur les 21 Labres parasités nous avons recueilli 187 Microcotyle donavini ce qui donne une moyenne de 9 parasites par hôte.

La répartition semble indiquer que le côté gauche est plus atteint que le droit ; en effet, nous avons trouvé :

- 113 Microcotyle sur les branchies du côté gauche;

- 74 Microcotyle sur les branchies du côté droit.

Nous avons en outre compté le nombre de parasites par arc en notant pour chacun la place sur l'arc lui-même. Cette répartition peut se résumer dans le tableau suivant :

\begin{tabular}{|c|c|c|c|}
\hline & & Côté gauche & Côté droit \\
\hline \multirow{2}{*}{$1^{\text {er }} A r c$} & Supérieur & 50 & 38 \\
\hline & Inférieur & 12 & 6 \\
\hline \multirow{2}{*}{$2^{e} A r C$} & Supérieur & 41 & 21 \\
\hline & Inférieur & 4 & 3 \\
\hline \multirow{2}{*}{$3^{e} A r c$} & Supérieur & 4 & 4 \\
\hline & Inférieur & 2 & 2 \\
\hline \multirow{2}{*}{$4^{\circ} A r c$} & Supérieur & 0 & 0 \\
\hline & Inférieur & 0 & 0 \\
\hline
\end{tabular}

Nous voyons immédiatement que le premier et le deuxième arc sont les plus parasités avec une prédominance pour le premier. Le troisième arc présente quelquefois des parasites ; le quatrième jamais.

Microcotyle donavini est générlement localisé à la partie supérieure du premier et du deuxième arc.

\section{Bibliographie}

Bychowsky (B. E.), 1957. - Systématique et phyllogénie des Trématodes Monogènes (en russe). Pub. Lab. Zool. Acad. Sc. U.R.S.S., Léningrad, 509 p. 315 fig.

Euzet (L.), 1957. - Larves gyrodactyloïdes nageantes de quelques Microcotylidae. (Trematoda. Monogenea). Bull. Soc. Neuch. Sc. Nat., 80, p. 187-194, fig. 1-24.

-, 1957. - Recherches sur les Monogenoïdea parasites de poissons marins. Ann. Parasit., 32 (5-6), p. $469-481$, fig. 1-17. 
—, 1958. - Sur le développement post-larvaire de Microcotylidae (Monogenoüdea Polyopisthocotylea). Bull. Soc. Neuch. Sc. Nat., 81, p. 79-84, fig. 1-7.

Llewellyn (J.) et OWen (I. L.), 1960. - The attachment of the monogenean Discocotyle sagittata Leuckart to the gills of Salmo trutta L. Parasitology, 50, p. 51-59, fig. 1-4, $1 \mathrm{pl}$.

ReMLeY (L. W.), 1942. - Morphology and life history studies of Microcotyle spinicirrus Mac Callum, 1918 a Monogenetic Trematode parasitic on the gills of Aplodinotus grunniens. Trans. Amer. Micr. Soc. 61, p. 141-155, fig. 1-14.

ScoTt (T.), 1905. - Observations on some parasites new or rare in Scottish waters. 23, rd. Rep. Fish. Bd. Scot. (3) Sci. Invest. p. 108-119 fig.

Sproston (N. G.), 1946. - A synopsis of the monogenetic Trematodes. Trans. Zool. Soc. Lond. 25, p. 185-600, fig. 1-118.

Van Beneden (P. J.) et Hesse (C. E.), 1863. - Recherches sur les Bdellodes ou Hirudinés et les Trématodes marins. Mém. Acad. Roy. Sc. Belgique, 34, p. 1-142, pl. 1-13.

Station biologique de Sète et C.S.U. Perpignan 